Laser Chem. 1988, Vol. 8, pp. 243-257

(C) 1988 Harwood Academic Publishers GmbH

Photocopying permitted by license only

Reprints available directly from the Publisher

Printed in the United Kingdom

\title{
Transient Isomerisation and Inverse Electronic Relaxation of Infrared Multiple-Photon Excited Pentafluoropyridine
}

\author{
P. K. CHOWDHURY, K. V. S. RAMA RAO and J. P. MITTAL \\ Chemistry Division, Bhabha Atomic Research Centre, \\ Trombay, Bombay-400 085, India
}

(Received August 24, 1987; accepted October 5, 1987)

Pentafluoropyridine (PFP) undergoes fast structural isomerisation to fulvenes when irradiated with $9 \mathrm{R}(16) \mathrm{CO}_{2}$ laser line in the fluence range $0.5-1.5 \mathrm{~J} / \mathrm{cm}^{2}$. The unstable fulvenes slowly decay back to PFP in ms time scale. No detectable permanent dissociation of PFP was observed in the above low fluence experiments. However, by using focused $\mathrm{CO}_{2}$ laser beam for the irradiation of PFP, an emission of light in the visible/near-UV was observed. The time evolution of the luminescence reveals three peaks at 390,460 and $500 \mathrm{~nm}$ on a broad background emission of $300-680 \mathrm{~nm}$. These bands are assigned to the fluorescence of PFP via inverse electronic relaxation (IER) and $\mathrm{C}_{2}$ Swan bands. On prolonged irradiation of PFP in focused condition a small extent of permanent dissociation was observed with the major products as $\mathrm{C}_{2} \mathrm{~F}_{4}$, a sooty yellow deposit and another compound presumed to be Dewar PFP.

KEY WORDS: Inverse electronic relaxation, IER, infrared multiple-photon excitation, IRMPE, structural isomerisation.

The radiationless internal conversion of electronic energy to the ground electronic state vibrational energy in isolated molecules has been used as a method of preparing vibrationally excited molecules. ${ }^{1,2}$ In contrast, strong vibrational excitation of a molecule by means of pulsed IR laser may induce internal energy transfer to the molecule's electronic excited state. Such a process is usually followed by emission of light in the visible or UV. This has become a current topic of active 
experimental and theoretical investigation. ${ }^{3-6}$ This process, known as inverse electronic relaxation (IER), has become feasible with the development of IR multiple-photon excitation (IRMPE) as a means of fast vibrational excitation of molecules. The multiphoton level of excitation is limited by the competitive rates of up-pumping and energy dependent molecular dissociation in the gound electronic state. If a suitable electronic excited state of a molecule is lying below its dissociation limit, visible/UV luminescence via IER could be observed. The pentafluoropyridine (PFP) was chosen for this study because of its high thermal stability and having fluorescence in the longer wavelength region $\left(\lambda_{\max }=405 \mathrm{~nm}\right)$.

Recently there are studies on the photophysics and photochemical isomerisation reactions of benzene, pyridine and their perfluoroanalogues. ${ }^{7,8}$ The formation of Dewar-type isomer is known for hexafluorobenzene, pyridine and pentafluoropyridine (PFP) by ultraviolet excitation. ${ }^{8,9,10}$. Recently Ratajczak et al. have reported ${ }^{8}$ that the UV flash photolysis of PFP in gas phase yields two transients which decay completely back to the original species in ms time scale. Their evidence from UV flash absorption spectroscopy and mass spectroscopy characterised the intermediates to be two fulvene isomers of PFP presumably formed via excited singlet and triplet. However, the time resolved studies in UV flash photolysis of benzene have indicated that internal conversion to vibrationally highly excited ground electronic state molecules, "hot molecules," is an intermediate step in the mechanism for the formation of fulvene isomer. ${ }^{11}$

We report that the formation of unstable fulvene isomers of PFP occurs by irradiation with a pulsed $\mathrm{CO}_{2}$ laser at a fluence of 0.5 to 1.5 $\mathrm{J} / \mathrm{cm}^{2}$. The transient fulvene isomers were found to revert back to the parent PFP in millisecond time scale. The same isomers of PFP were also observed in IR multiple-photon excited $\mathrm{SF}_{6}$ sensitised experiments. These results indicate that the structural isomerisation of aromatic molecules occurs in the ground electronic state. However, irradiating PFP by a focused $\mathrm{CO}_{2}$ laser beam results in emission of light in the visible and UV.

\section{EXPERIMENTAL}

A grating-tuned multimode TEA pulsed $\mathrm{CO}_{2}$-laser (Lambda Physik EMG-201E- $\mathrm{CO}_{2}$ ) was used. A typical pulse consisted of an intense 100 
ns spike followed by a very weak tail of $500 \mathrm{~ns}$ when the laser gas mixture was nitrogen free. The tail contains only $5 \%$ of the total energy in the pulse. The experimental details are as described in reference 12 .

A conventional greaseless glass vacuum system was used for sample preparation. All the fluoro-compounds used in this work were supplied by PCR Research Chemicals Inc. (Gainesville, Florida, USA). An indigenously built gas chromatograph ${ }^{13}$ with a flame ionisation detector was employed for gas analysis. The progress of the laser induced decomposition of PFP was monitored at $982 \mathrm{~cm}^{-1}$ with a Perkin-Elmer Model 577 Infrared grating spectrophotometer.

The time resolved experiments were carried out in a specially designed stainless steel cell of $290 \mathrm{~cm}^{3}$ volume. The cell was equipped with two pairs of orthogonal windows. One pair $\mathrm{KCl}$ windows were positioned $3 \mathrm{~cm}$ apart for $\mathrm{CO}_{2}$ laser irradiation and the other pair quartz windows $9 \mathrm{~cm}$ apart was used for monitoring $\mathrm{UV}$ beam as shown in Figure 1. A uniform $\mathrm{CO}_{2}$ laser beam with energy fluence between 0.5 and $1.5 \mathrm{~J} / \mathrm{cm}^{2}$ was used for the irradiation of pentafluoropyridine (PFP). A cw-spectroscopic UV-probe beam from a xenon lamp (150 W) with associated optics, monochromator $(f=3.5)$, fast PMT (1P

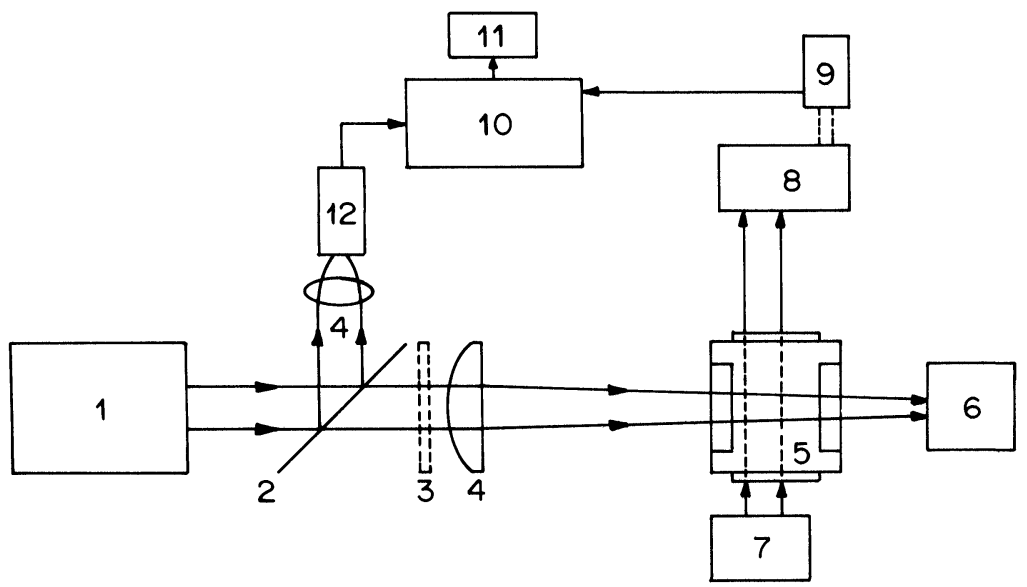

Figure 1 Schematic diagram of the experimental set-up for the transient absorption studies: 1-TEA $\mathrm{CO}_{2}$ laser, 2-Beam splitter, 3-Attenuator, 4- $\mathrm{BaF}_{2}$ lens, 5Stainless steel cell, 6-Energy meter, 7-Xenon lamp, 8-Monochromator, 9-PMT, 10-Biomation digitiser/averager, 11-Y-T Plotter, 12-Photon drag detector. 
28) and signal processing system (Biomation 4500) was used for the detection of transients produced in the $\mathrm{CO}_{2}$ laser irradiation. The Gould Biomation 4500 transient digitiser was triggered by the photon drag detector (Rofin Model 7415) signal generated by the partially reflected laser beam. A nearly parallel beam geometry was maintained in the sample cell by condensing the laser beam with a $f=100 \mathrm{~cm} \mathrm{BaF}_{2}$ lens and positioning the $3 \mathrm{~cm}$ IR optical pathlength of the cell between the lens and its focal point.

In some of the experiments the focused $\mathrm{CO}_{2}$ laser beam was used for irradiation. The focused irradiation geometry was maintained in the above mentioned cell by interchanging the IR and UV transparent windows to 9 and $3 \mathrm{~cm}$ respectively and by using a $f=10 \mathrm{~cm} \mathrm{BaF}{ }_{2}$ lens before the entrance $\mathrm{KCl}$ window. During monitoring emission the $\mathrm{Xe}$ lamp was put off.

In some experiments a $\mathrm{KrF}$ excimer laser $(248 \mathrm{~nm}$; full width at half-maximum, $12 \mathrm{~ns} ; 100 \mathrm{~mJ}$ ) from Oxford Lasers (UK) was used, instead of the $\mathrm{CO}_{2}$ laser, for the studies of transient detection by both absorption and emission method as mentioned above. The steady state fluorescence of PFP was recorded with a spectrofluorimeter (Aminco Model No 4-8202 B, USA) and a single photon counting fluorimeter (Model No 199 F, Edinburgh Instruments, UK) was used for the excited state lifetime measurement of PFP in the gas phase.

\section{RESULTS}

The infrared absorption spectrum of pentafluoropyridine (PFP) reveals two strong bands ${ }^{14}$ at $982 \mathrm{~cm}^{-1}$ and $1076 \mathrm{~cm}^{-1}$ corresponding to the output of the $\mathrm{CO}_{2}$ laser. The infrared spectrum of the gas after irradiation with $9 \mathrm{R}(16) \mathrm{CO}_{2}$ laser line in focused condition has generated new absorption peaks at 1186, 1332 and $1342 \mathrm{~cm}^{-1}$. The IR absorption peaks are due to tetrafluoroethylene (TFE) formed as a result of $\mathrm{CO}_{2}$ laser irradiation of PFP. However, when the $\mathrm{CO}_{2}$ laser irradiation of PFP was carried out in presence of excess of chlorine, $\mathrm{CF}_{2} \mathrm{Cl}_{2}$ was detected as a product with no $\mathrm{C}_{2} \mathrm{~F}_{4} \cdot{ }^{13}$ This indicates that $: \mathrm{CF}_{2}$ is formed as a primary product in this reaction.

The gas chromatographic analysis of the $\mathrm{CO}_{2}$ laser irradiation mixture gave three intense peaks two of which were characterised as $\mathrm{C}_{2} \mathrm{~F}_{4}$ and unreacted PFP respectively. The third one has been assigned 
to the Dewar isomer of PFP by comparing results from other laboratories. ${ }^{8,15}$ Two other minor products $\mathrm{C}_{3} \mathrm{~F}_{6}$ and $\mathrm{C}_{4} \mathrm{~F}_{8}$ and a sooty yellow polymer were also obtained.

\section{Time resolved absorption}

Figures 2 and 3 show an initial fast growth of optical absorption at 230 $\mathrm{nm}$ and $280 \mathrm{~nm}$ on pulsed $\mathrm{CO}_{2}$ laser irradiation of PFP, in a parallel beam geometry, at a fluence of $1 \mathrm{~J} / \mathrm{cm}^{2}$. The time resolved spectra of the fast rising signal has an optical absorption in the UV region with a maximum at $275 \mathrm{~nm}$, as shown in Figure 4. The initial absorption decays within a few tens of microseconds giving long lived absorption bands due to two transient species. In the region $230 \mathrm{~nm}$ to $265 \mathrm{~nm}$ a decrease in absorption compared with the PFP absorption is observed, whereas in the region 265 to $285 \mathrm{~nm}$ an increase is observed. During prolonged times of observation, millisecond time scale, the long lived transients decay (Figures 5 and 6 ) and the absorption spectrum of the

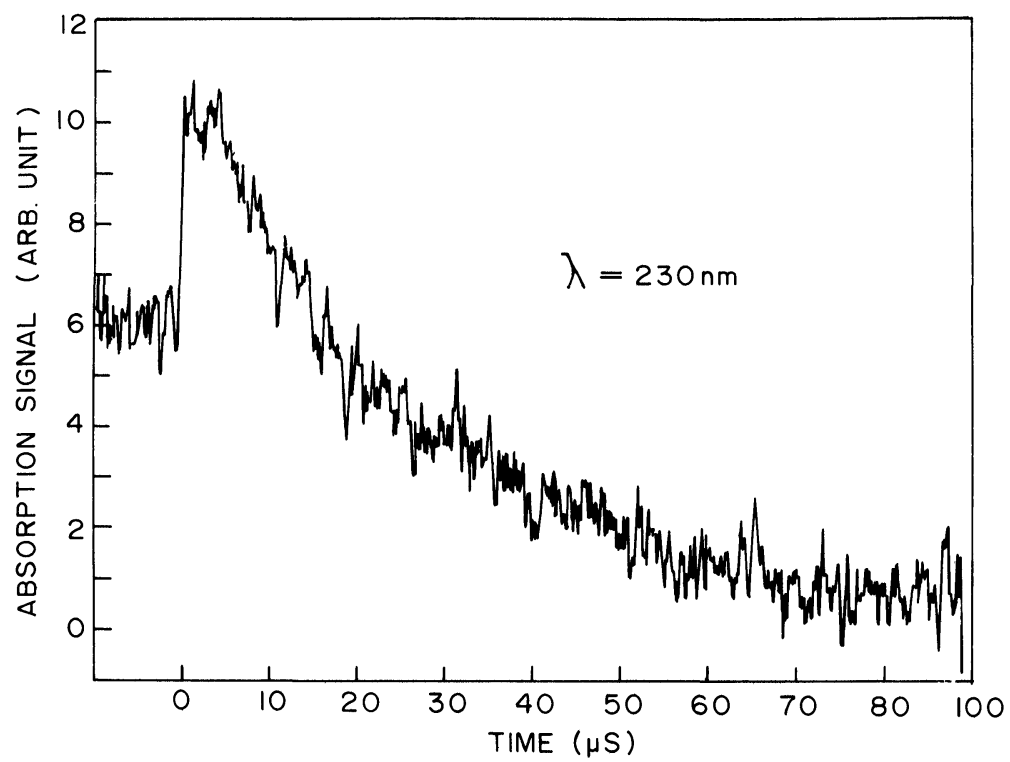

Figure 2 Growth and decay of the transient at $230 \mathrm{~nm}$ in $\mu$ s time scale. The $9 \mathrm{R}(16) \mathrm{CO}_{2}$ laser line was used at a fluence of $1 \mathrm{~J} / \mathrm{cm}^{2}$ to irradiate 3 torr of PFP. 


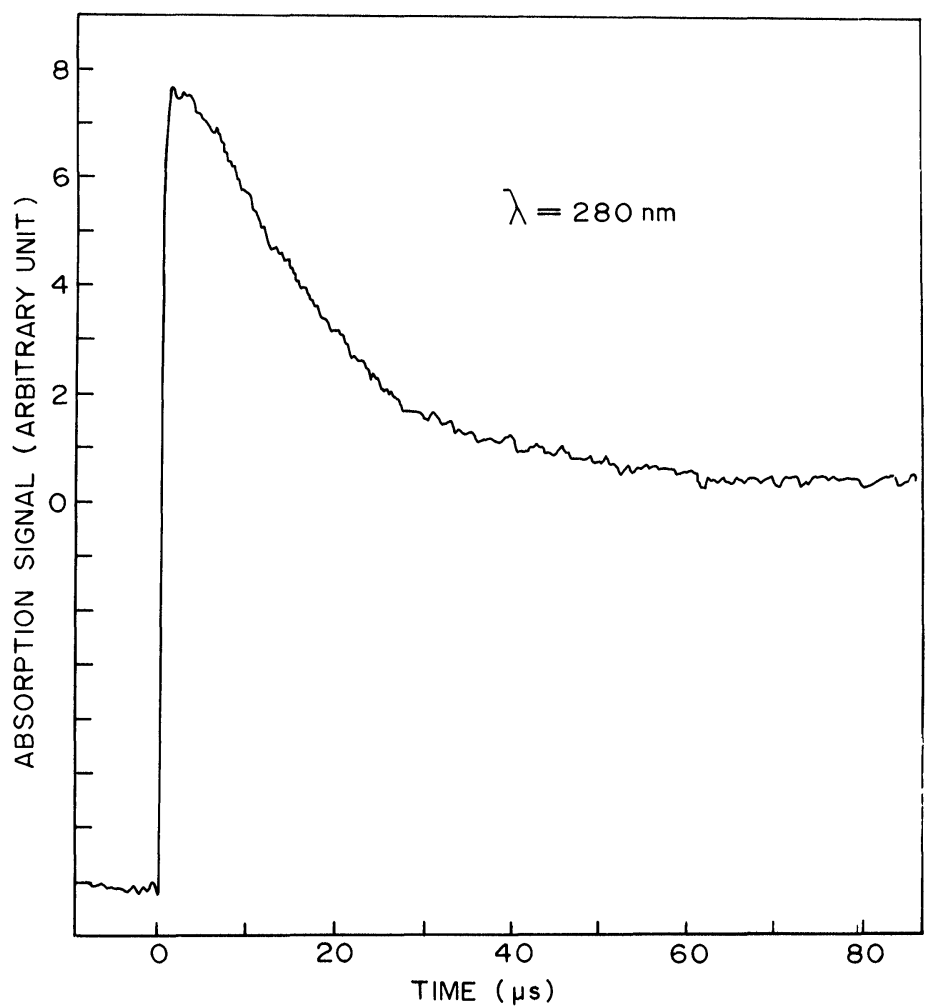

Figure 3 Growth and decay of the transient at $280 \mathrm{~nm}$, in the same condition as in Figure 2.

irradiated sample gradually returns to that of the original PFP. No stable photoproducts were detected in these parallel beam irradiations up to a laser fluence of $1.5 \mathrm{~J} / \mathrm{cm}^{2}$.

\section{Sensitised experiments}

When a mixture of 4 torr PFP and 8 torr $\mathrm{SF}_{6}$ was irradiated by the parallel beam of the $\mathrm{CO}_{2}$ laser up to $1.5 \mathrm{~J} / \mathrm{cm}^{2}$, no decomposition of PFP or $\mathrm{SF}_{6}$ was observed. However, time resolved experiments revealed the formation of above mentioned unstable transient species absorbing at 230 and $280 \mathrm{~nm}$. The transient species were observed by irradiating both the PFP and $\mathrm{SF}_{6}$ at the $\mathrm{CO}_{2}$ laser output tuned to $9 R(16)$ and $10 P(20)$ respectively. 


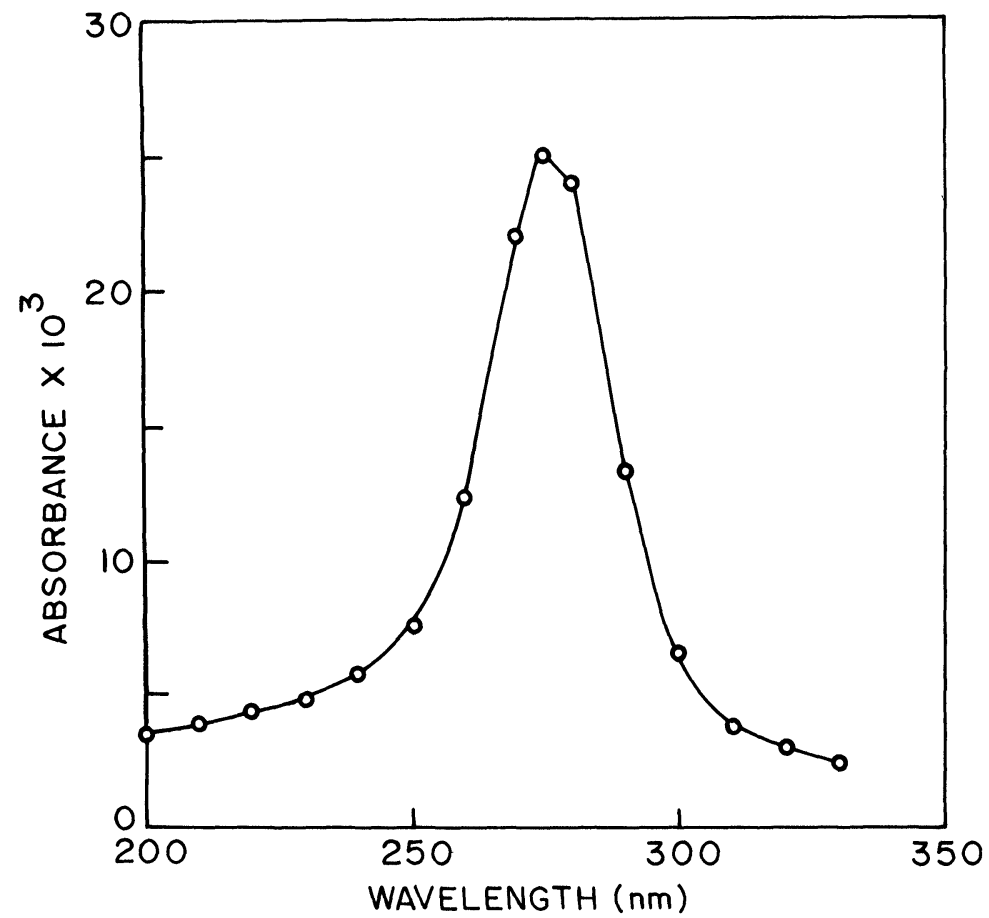

Figure 4 The time resolved absorption spectra of the initial transient formed at $1.5 \mu \mathrm{s}$ after the $\mathrm{CO}_{2}$ laser pulse.

\section{Excimer laser photolysis}

Similar formation of short and long lived transient species was observed by irradiation with $\mathrm{KrF}$ laser at $248 \mathrm{~nm}$. An emission at 400 $\mathrm{nm}$ was also monitored which appears to follow the $\mathrm{KrF}$ laser pulse shape.

\section{IR laser induced UV/VIS emission}

When the $\mathrm{CO}_{2}$ laser fluence was increased to $2 \mathrm{~J} / \mathrm{cm}^{2}$ and above for the irradiation of PFP, a sooty yellow deposit was observed on the optical windows which causes a glow in the subsequent pulses. To avoid such deposits on windows, a focused geometry was used so that the fluence 


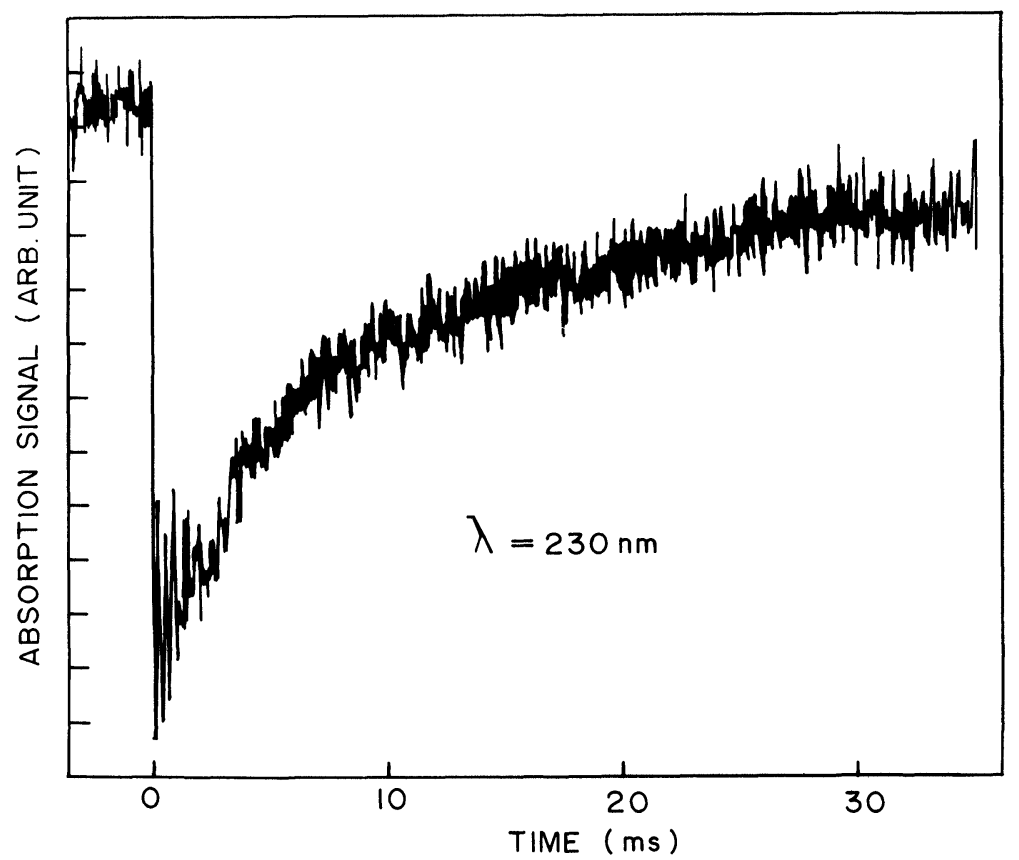

Figure 5 Decay of the transient absorption at $230 \mathrm{~nm}$ in $\mathrm{ms}$ time scale.

at the windows was low and the high fluence reaction volume was small. When the sample was irradiated at $1076 \mathrm{~cm}^{-1}$, there appeared a whitish blue light emission, from within the beam, at each laser pulse. The luminescene spectra recorded $400 \mathrm{~ns}$ after the $\mathrm{CO}_{2}$ laser pulse is shown in Figure 7. The spectra exhibits three peaks at 390, 460 and 500 $\mathrm{nm}$ respectively. The steady state broad fluorescene spectra of PFP at the excitation wavelength of $260 \mathrm{~nm}$ is also shown in the same Figure. The time dependence of the luminescene at 380 and $520 \mathrm{~nm}$ is shown in Figure 8.

\section{DISCUSSION}

The level of vibrational excitation of molecules depends in the IR laser fluence. While relatively low levels are populated in case of parallel beam irradiation with fluence of $0.5-2 \mathrm{~J} / \mathrm{cm}^{2}$, much higher multiphoton 


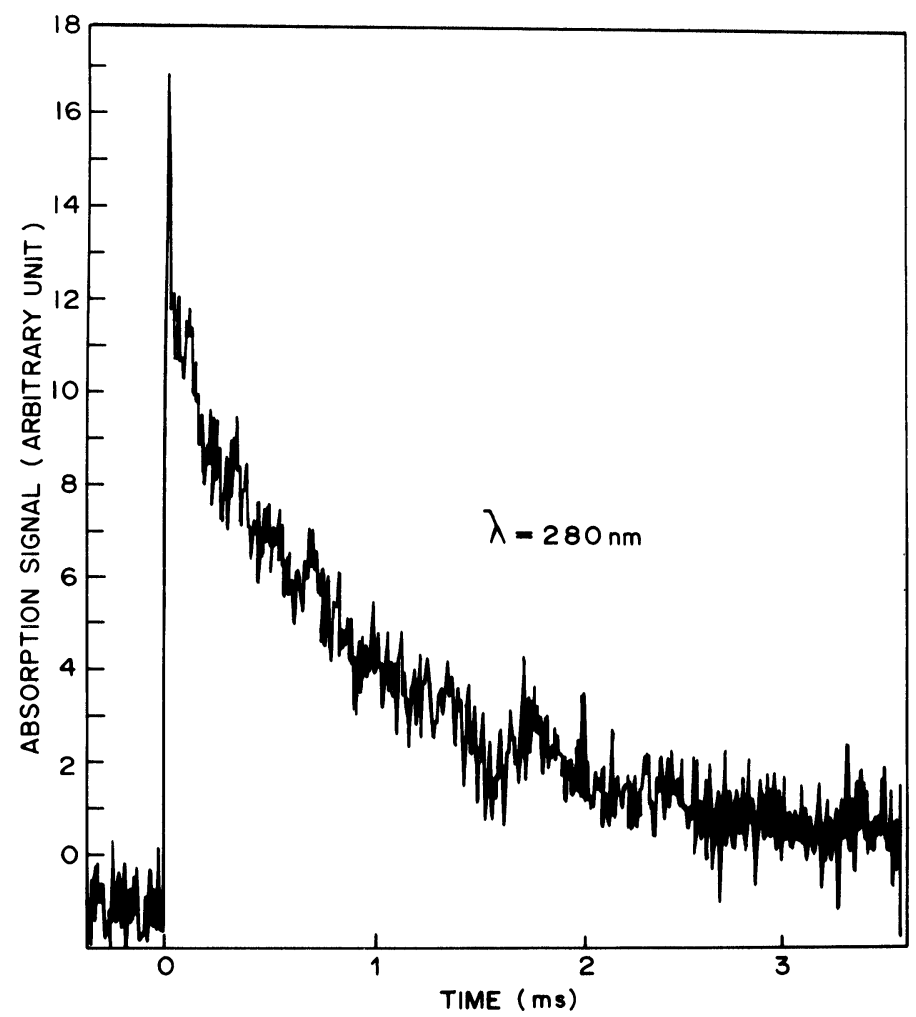

Figure 6 Decay of the transient absorption at $280 \mathrm{~nm}$ in ms time scale.

levels of excitation could be achieved by focusing the laser beam within the sample with focal fluence in the range $10^{2}-10^{3} \mathrm{~J} / \mathrm{cm}^{2}$. Permanent decomposition of PFP was observed only when a focused $\mathrm{CO}_{2}$ laser beam was used for irradiation. IR and GC analysis of the irradiated samples showed that the major decomposition product was $\mathrm{C}_{2} \mathrm{~F}_{4}$ which is formed by dimerisation of the primary photoproduct $: \mathrm{CF}_{2}$. The formation of : $\mathrm{CF}_{2}$ has also been observed by Hack and Langel ${ }^{16}$ in their $\mathrm{KrF}$ laser irradiation of hexafluorobenzene. In case of focused irradiation an intense visible/UV luminescence was also observed.

Formation of both short lived and long lived unstable transients were observed in the fluence range of $0.5-1.5 \mathrm{~J} / \mathrm{cm}^{2}$. Kinetic analysis of the growth and decay of the short lived transient showed that it is 


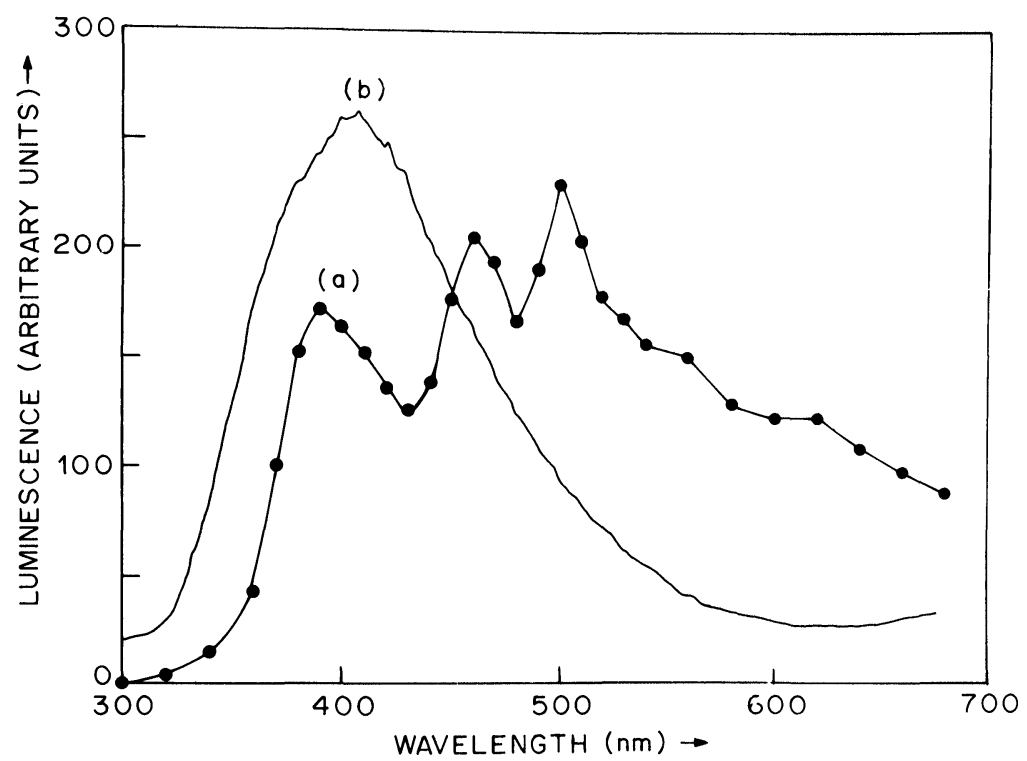

Figure 7 Time resolved emission spectra at $400 \mathrm{~ns}$ after the $\mathrm{CO}_{2}$ laser pulse in focused geometry of irradiation. The laser energy per pulse was kept at $200 \mathrm{~mJ}$ with the $9 \mathrm{R}(16)$ $\mathrm{CO}_{2}$ laser line.

formed with a rate constant $\mathrm{K}_{f}=3 \times 10^{6} \mathrm{~s}^{-1}$ and decayed with $k_{d}=3.6 \times 10^{4} \mathrm{~s}^{-1}(230 \mathrm{~nm})$ and $5.8 \times 10^{4} \mathrm{~s}^{-1}(280 \mathrm{~nm})$.

It is clear from the above results that two transients, one absorbing in the region 230-265 $\mathrm{nm}$ and the other absorbing in the region $265-285$ $\mathrm{nm}$, are produced by both the $\mathrm{KrF}$ laser and $\mathrm{CO}_{2}$ laser irradiations of PFP. Both the transients disappear in several milliseconds after the laser pulse, reverting back to PFP. No stable photoproducts were detected in any of these low influence experiments. The lifetime of the transients were calculated to be $\tau_{1}=15 \mathrm{~ms}$ and $\tau_{2}=1 \mathrm{~ms}$ respectively when measured at 3 torr of PFP. On the basis of the mass spectral evidence the transients were characterised by Ratajczak ${ }^{8}$ as the two fulvene isomers of PFP:<smiles></smiles>

and<smiles></smiles> 


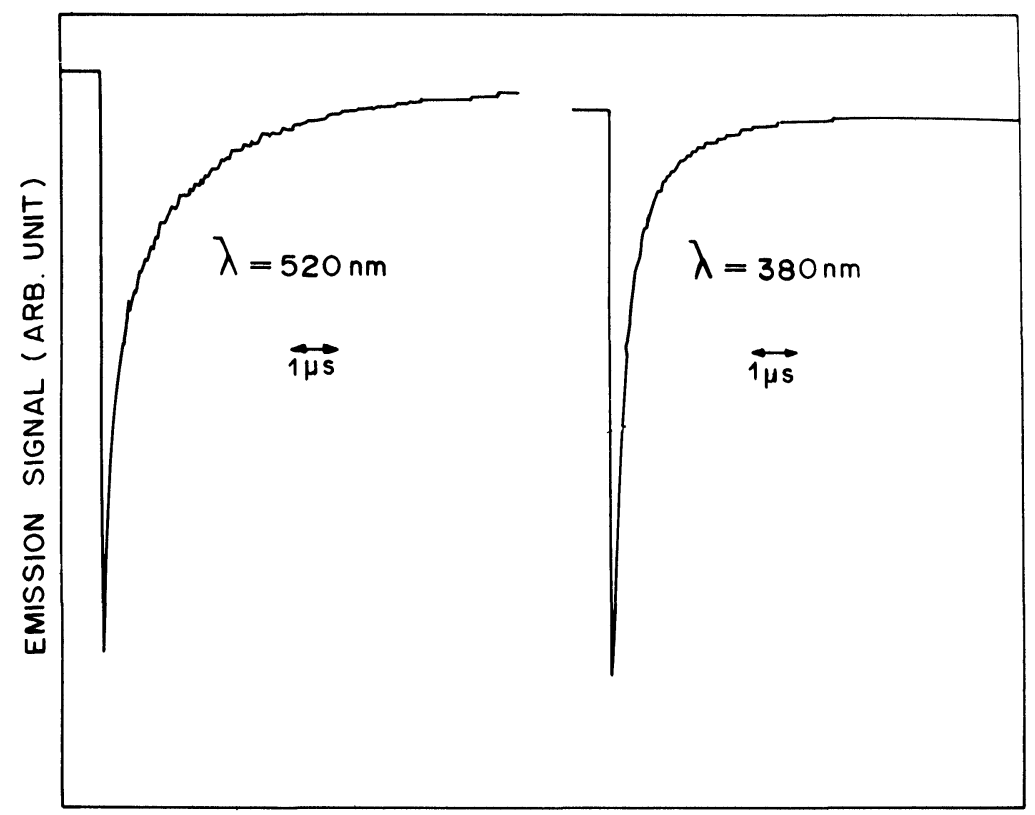

Figure 8 Growth and decay of emission at $380 \mathrm{~nm}$ and $520 \mathrm{~nm}$ in $\mu$ s time scale.

They have indicated that these fulvene isomers are possibly formed from the electronically excited singlet or triplet PFP molecules. The fact that no emission was observed at a fluence of $1 \mathrm{~J} / \mathrm{cm}^{2}$ used in our experiment rules out population of singlet electronic excited states by inverse relaxation from these relatively low vibrational levels accessible at these low fluences. In as much as this is the case, we conclude that the isomerisation of PFP to fulvenes proceeds via vibrational excitation only without any involvement of singlet excited state.

The initial transient formed with a peak optical absorption at $275 \mathrm{~nm}$ is the nascent vibrationally excited PFP which undergoes structural change in $\mu$ s time scale to form two fulvene isomers of PFP. The same optically characterised transient was formed in $\mathrm{KrF}$ excimer laser irradiation. At high temperature the fulvene structure could be thermodynamically more stable. ${ }^{17}$ This is assumed because in our studies 
the vibrationally excited state of PFP has been prepared by $\mathrm{CO}_{2}$ laser excitation, by energy transfer from IR multiphoton excited $\mathrm{SF}_{6}$ and by $\mathrm{KrF}$ laser excitation. In the latter case the vibrational excitation is induced by internal conversion of the singlet excited state by nonradiative relaxation. As the system cools down in a few tens of ms the fulvenes revert back to the PFP, the thermodynamically more stable structure at room temperature. The rise and decay of the vibrationally excited state of PFP, which is the transient intermediate for the formation of the fulvene, has been studied. The fast rise of the transient absorption signal was observed in case of $\mathrm{CO}_{2}$ laser and $\mathrm{KrF}$ laser excitation. Whereas the rise was observed to be slow and it varies with the $\mathrm{CO}_{2}$ laser pulse shape and the $\mathrm{SF}_{6}$ gas pressure in case of sensitised excitation by $\mathrm{SF}_{6}$. It would be interesting to see whether only PFP* luminescence could be observed at moderate $\mathrm{CO}_{2}$ laser fluence of 2 to $10 \mathrm{~J} / \mathrm{cm}^{2}$. Unfortunately, we could not spectrally resolve the luminescence observed at these moderate fluence. We are planning to have an in-depth understanding of IER and associated processes such as product fragments emission by further time resolved experiments.

\section{Luminescence}

In the focused irradiation of PFP by a pulsed $\mathrm{CO}_{2}$ laser, an emission of light in the visible/UV was observed. That the light was generated from the multiphoton excited PFP has been verified by tuning the $\mathrm{CO}_{2}$ laser to four different laser lines at $9 \mathrm{R}(16), 9 \mathrm{P}(26), 10 \mathrm{R}(30)$ and $10 \mathrm{P}(20)$ with the same energy per pulse. While emission of light was observed for the first three laser lines, there was no emission from the same for irradiation at $10 \mathrm{P}(20)$ laser line which is off-resonant from the molecular absorption modes. From the observed short wavelength cutoff of the emission spectrum, we find IRMPE level of PFP is at least equivalent to some 35 laser photons.

The rise and decay of the visible/UV light was monitored to be very fast. The rise time of the emission was found to be faster than the rise of the transient absorption at $275 \mathrm{~nm}$ and the emission signal decays to the base line in a few $\mu$ s. The time resolved spectra of the emission at $400 \mathrm{~ns}$ (Figure 7) shows 3 peaks at 390, 460 and $500 \mathrm{~nm}$. For comparison the steady state fluorescence of the PFP at the same sample pressure has also been recorded and given in the same Figure 7. The $390 \mathrm{~nm}$ peak could be due to the IR laser induced fluorescence from 
the PFP molecule. The fluorescence life time of PFP is less than $200 \mathrm{ps}$ as measured with the single photon counting fluorimeter. However, the $15 \mathrm{~nm}$ blue shift in the emission could be due to a slightly different vibronic level population in the inverse electronic relaxation of IR multiphoton excitation of PFP. The criteria for observing IER ${ }^{6,18}$ in a molecule is that the lowest emitting singlet state should be below the dissociation level D of the molecule and the $\rho_{s} / \rho_{g}$ is not too small. The $\rho_{s}$ and $\rho_{g}$ are the vibrational level density of the first singlet emitting state and the ground electronic state respectively. If the dissociating level is close to the emitting state, a competitive process of unimolecular dissociation with its rate,

$$
k(E)=A\left[\frac{E-D}{E}\right]^{s-1} \sec ^{-1}
$$

occurs, where $A$ is a constant, $E$ is the total vibrational energy of the molecule and $S$ is the number of vibrational degrees of freedom. For intermediate size molecule $(S=10)$ the unimolecular decay does not allow the molecule to be overexcited considerably and hence it can not reach high-lying electronic state. Besides, in the accessible energy range $(E-D)$ over the dissociation limit such decay effectively competes with IER. Molecules with a large number of vibrational degrees of freedom, $S=27$ for PFP, the molecule can be greatly excited over the dissociation limit. For this molecule a range of $(E-D)$ with a small value of $k(E)$ becomes a reality and in this case the emitting state may even lie over the dissociation limit within $(E-D)$.

The laser induced emission spectrum consisted of two additional peaks at 460 and $500 \mathrm{~nm}$ which are not yet fully understood. By comparing with literature, these peaks could be due to $C_{2}$ Swan bands ${ }^{19}$. The emission of $\mathrm{C}_{2}{ }^{*}$ has been occasionally observed ${ }^{20}$ in the focused $\mathrm{CO}_{2}$ laser irradiation of organic molecules.

\section{CONCLUSION}

On pulsed $\mathrm{CO}_{2}$ laser irradiation, PFP reveals very interesting behaviour depending on the laser energy fluence. At a moderate laser 
energy fluence of $0.5-1.5 \mathrm{~J} / \mathrm{cm}^{2}$, PFP undergoes structural isomerisation to form unstable fulvenes which revert back to the parent molecule in millisecond time scale. The same fulvenes were also observed in the $\mathrm{SF}_{6}$ sensitised experiments following the $\mathrm{CO}_{2}$ laser excitation of the sensitiser. No detectable dissociation of PFP was observed in the above experiments. However, on focused irradiation of PFP, emission of visible/UV light accompanied by a very small dissociation was observed. The time evolution of the luminescence reveals three peaks at 390,460 and $500 \mathrm{~nm}$ on a broad background emission of $300-680 \mathrm{~nm}$. These are assigned to the fluorescence of PFP via inverse electronic relaxation (IER) and $\mathrm{C}_{2}$ Swan bands respectively. The major dissociation products characterised were $\mathrm{C}_{2} \mathrm{~F}_{4}$, a sooty yellow deposit and another compound assigned to be Dewar PFP.

\section{Acknowledgements}

The authors are grateful to Dr. R. M. Iyer, Director, Chemical Group, for his keen interest in this work. They thank Mr. K. A. Rao for his help in GC analysis.

\section{References}

1. D. F. Heller and G. A. West, Chem. Phys. Lett. 69, 419 (1980).

2. J. E. Dove, H. Hippler and J. Troe, J. Chem. Phys. 82, 1907 (1985).

3. A. Nitzan and J. Jortner, J. Chem. Phys. 71, 3524 (1979).

4. J. W. Hudgens, J. L. Durant, Jr., D. J. Bogan and R. A. Coveleskie, J. Chem. Phys. 70, 5906 (1979).

5. A. A. Makarov, G. N. Makarov, A. A. Puretzky, V. V. Tyakht, Appl. Phys. 23, 391 (1980).

6. A. A. Puretzky, Laser Chem. 6, 103 (1986).

7. C. S. Permenter, Advan. Chem. Phys. 22, 365 (1972); L. D. Zeigler and B. S. Hudson, Excited States, Ed. E. C. Lim, Acad. Press, New York, (1982), Volume 5, pp. 41-140.

8. E. Ratajczak and B. Sztuba, J. Photochem. 13, 242 (1980).

9. I. Haller, J. Chem. Phys. 47, 1117 (1967).

10. K. E. Wilzback and D. J. Rausch, J. Amer. Chem. Soc. 92, 2178 (1970).

11. N. Nakashima and K. Yoshihara, J. Chem. Phys. 79, 2727 (1983).

12. P. K. Chowdhury, K. V. S. Rama Rao and J. P. Mittal, J. Phys. Chem. (in press).

13. P. K. Chowdhury, K. V. S. Rama Rao and J. P. Mittal, J. Phys. Chem. 90, 2877 (1986).

14. D. A. Long and R. T. Bailey, Trans. Faraday Soc. 59, 599 (1963).

15. I. Haller, J. Amer. Chem. Soc. 88, $2070(1966)$.

16. W. Hack and W. Langel, J. Phys. Chem. 87, 3462 (1983). 
17. L. T. Scott and J. Maitland, Jr., Chem. Rev. 72, 181 (1972).

18. T. B. Simpson and N. Bloembergen, Chem. Phys. Lett. 100, 325 (1983).

19. K. P. Huber and G. Herzberg, Constants of Diatomic Molecules, (Von NostrandReinhold, Princeton, NJ, 1979), pp. 112-154.

20. R. V. Ambartzumian, N. V. Chekalin, V. S. Letokhov and E. A. Ryabov, Chem. Phys. Lett. 36, 301 (1975). 